\title{
Steps forward in LRTI and tuberculosis: update from the ERS Respiratory Infections
}

\section{Assembly}

\author{
F. Blasi*, R. Cosentini ${ }^{\#}$, G.B. Migliori ${ }^{\top}$ and W. Boersma ${ }^{+}$
}

ABSTRACT: Lower respiratory tract infections (LRTIs) and tuberculosis are among the leading reasons for seeking medical care.

In the present report, the most recent advances in the fields of clinical research and basic science of LRTIs and tuberculosis are presented through analysis of some of the best abstracts presented at the 18th European Respiratory Society Annual Congress in Berlin.

The role of viruses in chronic obstructive pulmonary disease exacerbations and the importance of new biomarkers in the diagnosis of bacterial infections in LRTI are discussed.

New tools for the diagnosis of latent and active tuberculosis in special subgroups of patients (children, immunocompromised individuals, etc.), and the new epidemiological threat of multidrug-resistant and extensively drug-resistant tuberculosis cases is analysed.

\section{KEYWORDS: European Respiratory Society Congress, respiratory infections, tuberculosis}

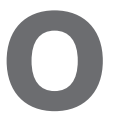
$\mathrm{n}$ the one hand, lower respiratory tract infections (LRTIs) are common and associated with major morbidity, mortality and financial burden [1]. On the other, tuberculosis (TB) has caused more deaths in the last hundred years than influenza and HIV combined, and millions of people die annually due to TB.

A number of important issues and challenges in the fields of clinical research and basic science remain open.

Drug-resistant TB has now emerged as a public health concern of major proportions, and the most alarming issue in international TB control is being faced, the emergence of extensively drugresistant (XDR) TB $[2,3]$.

The European Respiratory Society (ERS) Respiratory Infections Assembly actively supports research and educational initiatives in these areas, being also involved in European Union-funded protocols within the 6th and 7th Framework Programmes, e.g. the network of excellence, Genomics to combat Resistance against Antibiotics in Communityacquired LRTI in Europe (GRACE) [4], and the large-scale integrating project, Pan-European network for study and clinical management of drug resistant tuberculosis (TB PAN-NET), on TB.
The present article analyses some of the best abstracts presented at the 18th ERS Annual Congress in Berlin by members of the Respiratory Infections Assembly.

\section{RESPIRATORY INFECTIONS}

Of the 239 abstracts sent to the Respiratory Infections Group, 186 (78\%) were accepted for the ERS Annual Congress in Berlin. From these, three scientifically interesting abstracts were selected and are discussed below.

Exacerbations of chronic obstructive pulmonary disease (COPD) represent one of the major contributors to the clinical load of physicians, in both the hospital setting and the community.

Interest in viral infections as a cause of COPD exacerbations has been renewed since the introduction of PCR technology. With this technique, the most commonly identified viruses and their prevalence have been better identified in asthma and COPD patients. Respiratory viruses have been isolated in approximately half of exacerbations [5]. Rhinovirus can be recovered from induced sputum in up to $23 \%$ of COPD patients during exacerbation, but in $<1 \%$ of stable patients [6]. Exacerbations triggered by respiratory viruses are also more severe, associated with longer recovery times and have a greater chance
AFFILIATIONS

*Institute of Respiratory Diseases, University of Milan, Ospedale Maggiore Fondazione IRCCS Policlinico,

\#Emergency Medicine Dept, Ospedale Maggiore Fondazione IRCCS Policlinico, Mangiagalli e Regina Elena, Milan,

"World Health Organization Collaborating Centre for Tuberculosis and Lung Diseases, Fondazione S.

Maugeri, Care and Research Institute, Tradate, Italy.

+Medical Centre Alkmaar, Alkmaar, The Netherlands.

\section{CORRESPONDENCE}

F. Blasi

Istituto Malattie dell'Apparato Respiratorio, University of Milan, Padiglione Sacco Ospedale Maggiore Fondazione IRCCS Policlinico Mangiagalli e Regina Elena, Via F. Sforza 35, 20122 Milan, Italy Fax: 390250320628

E-mail: francesco.blasi@unimi.it

Received:

November 052008

Accepted after revision:

December 182008

STATEMENT OF INTEREST A statement of interest for $W$. Boersma can be found at www.erj.ersjournals.com/misc/ statements.dtl 
of hospital admission than exacerbations in which respiratory viruses were not detected [7].

In the abstract of ALBER et al. [8], entitled "Quantitative changes in rhinovirus load in exacerbations of COPD", the time course of viral load and nasal symptoms were investigated. Nasal symptoms were recorded and sputum samples collected before, during and 2 and 5 weeks after the exacerbation. Real-time PCR was used to quantify viral load. Rhinovirus was detected in a total of 111 $(29.7 \%)$ patients and at baseline in $12.3 \%$ (170 patients). The mean \pm SD viral load at baseline was $3.2 \times 10^{3} \pm 2.5 \times 10^{4}$ plaqueforming units $(\mathrm{pfu}) \cdot \mathrm{mL}$ sputum ${ }^{-1}$ and increased at exacerbation onset to $9.6 \times 10^{4} \pm 5.4 \times 10^{4} \mathrm{pfu} \cdot \mathrm{mL}^{-1} \quad(\mathrm{p}=0.008)$. There was a decline in viral load during follow-up. A higher rate of rhinovirus was detected if patients had rhinorrhoea and were sneezing. The viral load was highest in patients with symptoms such as rhinorrhoea $\left(1.6 \times 10^{5} \mathrm{pfu} \cdot \mathrm{mL}^{-1}\right)$ or sneezing $\left(1.1 \times 10^{6} \mathrm{pfu} \cdot \mathrm{mL}^{-1}\right)$ and significantly different from those without these symptoms. The authors concluded that viral load increased significantly on exacerbation and remained raised at 2 weeks. Moreover, a significant correlation was demonstrated between nasal symptoms and viral load.

Two interesting observations should be discussed. First, at baseline, a remarkably high rhinovirus load could be found in induced sputum, which might be explained by the hypothesis that a chronic infection with rhinovirus is present. Secondly, there was only a 30-fold increase in rhinovirus load during exacerbation, and this decreased to the viral load at baseline in 5 weeks. Only a third of the included patients at exacerbation were sampled at 5 weeks. The clearance of rhinovirus RNA should be studied in more detail.

Levels of biomarkers, especially procalcitonin (PCT), increase rapidly in serum in the presence of bacterial infection [9]. Therefore, bacterial infections may be differentiated from viral infections. An interesting development is so-called PCT-guided antibiotic therapy, which means that only patients with community-acquired pneumonia (CAP) or exacerbation of COPD with a high PCT level $\left(>0.25 \mu \mathrm{g} \cdot \mathrm{L}^{-1}\right)$ should be treated with antibiotics [10]. In a randomised study of COPD exacerbations, this method reduced antibiotic prescription by $32 \%$ without difference in outcome. These findings may have a great influence on antibiotic exposure in the near future.

In the study of LACOMA et al. [11], a number of biomarkers, such as PCT, neopterin, C-reactive protein (CRP) and midregional-pro-atrial natriuretic peptide (MR-pro-ANP), were measured in 286 serum samples from COPD patients at baseline, during exacerbation and in those with pneumonia. The questions of the study were as follows. 1) Are there any differences between the biomarkers among the three groups? 2) Can these biomarkers help in identifying exacerbations caused by bacteria? Serum samples $(n=286)$ from COPD patients were included; 14 samples were taken during a stable period (group 1), 217 during exacerbation (group 2) and 55 from patients with CAP (group 3). A significant relationship in PCT and CRP levels was found when the three groups were compared $(p<0.0001)$; the highest levels were detected in the CAP group. The highest concentrations of neopterin were found in patients with CAP and differed significantly from those in the other two groups. The levels of MR-pro-ANP did not differ in the three groups. When exacerbations of COPD with and without bacteria in sputum were studied in detail, no difference in CRP and PCT levels was found. Only neopterin and MR-pro-ANP were significantly different. From this study, it has been concluded that clinically stable COPD patients had lower levels of CRP, PCT and neopterin compared to an episode of exacerbation or CAP. No firm conclusion could be reached as to whether biomarkers are helpful in discriminating infectious from noninfectious exacerbations looking at bacteria in sputum.

This study confirmed that biomarkers, such as CRP and PCT, are helpful in moderate-to-severe infections. Neopterin levels are also raised in patients with CAP, but it is unknown what the added value is compared to the old markers CRP and PCT. The presence of bacteria in sputum at exacerbation and the role of biomarkers remains unresolved.

A very interesting abstract concerning Mycobacterium avium intracellulare complex (MAC) was submitted by MAEKURA et al. [12] from Japan. Since the mid-1990s, macrolide-containing regimens have been introduced in MAC pulmonary disease [13]. Sputum conversion with $\geqslant 12$ months' therapy can be observed in up to $90 \%$ of patients with lung disease [14]. However, information is scarce about the long-term effect of these macrolide-containing regimens. For patients who initially show sputum conversion (three consecutive acid-fastbacillus-negative cultures) while on medication but who subsequently develop cultures positive for MAC after discontinuing therapy, it has been shown that these patients can be re-infected by new MAC strains (genotypes) rather than manifesting disease due to relapse with their initial MAC strain (genotype) [15].

In the Japanese study, 5-yr survival and prognostic factors were retrospectively investigated in 399 patients with MAC pulmonary disease, of whom 76 died. The $50 \%$ survival rate was $16.3 \pm 0.5 \mathrm{yrs}$, with the highest survival in females $(89.8 \%)$. The prognosis in patients with cavitary lesions was significantly worse than in patients with bronchiectasis $(p<0.01)$. Patients who underwent clarithromycin-containing regimens (165 cases; 92.3\% survival) had a better outcome than other patients (234 cases; 82\% survival). Patients with MAC pulmonary disease diagnosed since 1997 had a better prognosis than those diagnosed before $1997(\mathrm{p}<0.05)$.

It was concluded that survival of MAC pulmonary disease has improved since 1997. In particular, females and patients with bronchiectasis are doing better.

This study, in a large group of patients, provides new information about the prognosis of MAC pulmonary disease in relation to macrolide-containing regimens. However, a number of issues in this disease, as stated in American Thoracic Society nontuberculous mycobacterial diseases guidelines, remain unresolved [16]. For instance, it is not known whether one drug (clarithromycin) is superior to another (azithromycin). No studies have compared two- versus threedrug regimens. What is the role of new-generation fluoroquinolones? What should be done with the patient who does not respond to a macrolide-containing treatment? In our opinion, MAC pulmonary disease may be underestimated. Clinicians 
should be aware of this disease, especially when underlying predisposing factors, such as pre-existing pulmonary disease or immune deficiency, are present. Chronic cough, dyspnoea, asthenia and weight loss are the most common symptoms. Treatment should be fine tuned according to radiographic features and the severity of nontuberculous mycobacterial disease.

\section{TUBERCULOSIS}

Among the large number (360) of abstracts accepted by the Tuberculosis Group ERS, several contributions from the main research groups within and outside Europe merit discussion.

Among others, the following hot topics attracted the researchers' interest: 1 ) the new interferon- $\gamma$ release assays (IGRAs); and 2) new findings concerning multi-drug resistant (MDR) TB and XDR-TB cases.

\section{IGRAs in diagnosis of latent TB infection and TB disease}

Research on IGRAs is currently focused on answering open questions where evidence is still lacking. Among the areas needing further evidence, mention should be made of the role and contribution of IGRAs in diagnosing latent TB infection (LTBI) in special populations (children, immunocompromised individuals, etc.), in diagnosing TB disease with the focus on difficult-to-diagnose disease (sputum-smear-negative and extrapulmonary $\mathrm{TB}$ ) and in monitoring the effectiveness of treatment of both LTBI and TB disease. Furthermore, several groups are looking for new biomarkers with a potential role (alternative or complementary) in improving the sensitivity and/or specificity of the existing QuantiFERON or enzymelinked immunospot (ELISPOT) IGRAs [17].

The abstracts adding new information to the existing body of knowledge are summarised below.

RD1-based tests for the diagnosis of TB disease or infection in specimens other than blood

Among RD1-based tests for diagnosis of TB disease or infection in specimens other than blood, the study of HINKS et al. [18], performed in India, provided proof of principle that antigen-specific T-cells are concentrated in the cerebrospinal fluid and that ELISPOT may represent a rapid, sensitive $(90 \%$ in the present pilot study) and specific (100\% in present study) test for TB meningitis, and the Tuberculosis Network (TB-NET) study [19] demonstrated that enumeration of M. tuberculosisspecific T-cells by ELISPOT has higher sensitivity than $M$. tuberculosis-specific PCR for the diagnosis of sputum-smearnegative TB.

Use of different RD1 antigen formats, such as peptides selected from intact RD1 proteins

The home-made interferon- $\gamma$ RD1-based assay tested by the TB-NET in seven countries demonstrated similar sensitivity and higher specificity than the tuberculin skin test (TST), QuantiFERON-TB Gold (QFT-G; Cellestis, Carnegie, Australia) and ELISPOT for diagnosis of TB disease [20]. The response to this test and QFT-G In-Tube over time is potentially useful in monitoring the effects of LTBI treatment in the paediatric population [21]. The same test also seems promising in the diagnosis of active sputum-smear-negative and extrapulmonary TB [22].
Detection of markers alternative or complementary to the IGRA Interferon- $\gamma$-inducible protein 10 (IP-10) and monocyte chemotactic protein- 2 are among the markers alternative or complementary to the IGRA. Since the diagnostic performances of IP-10 were satisfactory compared with those of QFT-G In-Tube [23], the authors concluded that the IP-10-based test may represent a new generation of sensitive tests characterised by simpler readout formats. The same group also demonstrated that both biomarkers are potentially able to improve sensitivity in immunocompromised patients [24].

\section{Different readouts for the detection of RD1 responses}

Fluorescence-activated cell sorting analysis and evaluation of long versus short assays are among the readouts for the detection of RD1 responses. The German-UK collaborative study [25] demonstrated that flow cytometric analysis of antigen-specific CD4 T-cell cytokine profiles is able to distinguish active TB from successfully treated disease. The study of CARRARA et al. [26] suggested that interferon $-\gamma$ memory response to RD1 proteins and other cytokines other than interferon- $\gamma$ ones (tumour necrosis factor (TNF)- $\alpha$ and interleukin-2) detected at day 1 (after in vitro stimulation) may improve identification of LTBI.

\section{Categories of patient in whom RD1 responses are evaluated Children}

According to the Modena group study results [27], younger children yielded higher rates of indeterminate results when tested with ELISA-based (QFT-G and QFT-G In-Tube) than with ELISPOT-based tests (T-SPOT.TB; Oxford Immunotec, Abingdon, UK). The role of T-SPOT.TB in diagnosing LTBI in bacille Calmette-Guérin (BCG)-vaccinated children is discussed in the study of the Riga group [28]. The Naples group study [29] demonstrated poor agreement among the TST, QFT-G In-Tube and T-SPOT.TB in a group of long-term pharmacologically immunosuppressed children due to rheumatological disease or liver transplantation.

\section{HIV-positive subjects}

The Spanish study [30] suggested that QFT-G In-Tube and TSPOT.TB exhibit similar results in HIV-positive subjects, the results being similar to TST when the CD4 count is $<250$ cells $\mu \mathrm{L}^{-1}$, and that both in vitro tests are less affected by BCG than the TST. The Ugandan study [31] suggested that the sensitivity of the TST, QFT-G In-Tube and T-SPOT.TB in the diagnosis of LTBI in patients with moderately advanced HIV infection seems to be unaffected by the degree of immunodeficiency, although the degree of concordant test results was relatively low.

\section{Pharmacologically immunocompromised patients}

The Italian study [32] suggested that QFT-G In-Tube can be used as a screening tool in pharmacologically immunocompromised patients under consideration for anti-TNF- $\alpha$ therapy due to the low number of indeterminate results. The Turkish study results [33] supported the use of QFT-G in diagnosing LTBI in these patients, although the number of indeterminate results was high.

\section{Healthcare workers}

Both the Italian study from Sondalo and Brescia (comparing TST and QFT-G in healthcare workers) [34] and the Indian study 
(comparing TST and QFT-G In-Tube in nursing students) [35] suggested poor agreement between these tests.

\section{Chronic patients undergoing haemodialysis}

The Swiss study [36] suggested higher sensitivity of TSPOT.TB and QFT-G In-Tube than TST in detecting LTBI, with moderate agreement of the two IGRAs and confirmation of their unreliability in detecting prior TB. The Japanese study [37] confirmed better results of QFT-G In-Tube over TST in detecting LTBI.

\section{Evaluation of the predictive value of RD1 in diagnosing TB} disease and comparison of performance of two commercially available RD1 tests

The results of the German group suggested that QFT-G InTube determines LTBI more accurately and provides a better indicator of progression from TB infection to TB disease than the TST [38]. The Tokyo group identified higher sensitivity and lower specificity of T-SPOT.TB versus QFT-G in the diagnosis of LTBI in Japanese subjects [39].

\section{MDR- and XDR-TB}

Although XDR-TB has been described only recently, more and more evidence is accumulating regarding this new form of TB [40]. The TB-NET provided a key contribution in demonstrating, for the first time, the clinical and prognostic differences between XDR- and MDR-TB, particularly in HIVnegative patients. Many public health questions still need an adequate answer, being mainly focused on how to prevent selection of resistant mutants. Of the key clinical research priorities, how to make better use the existing drugs while waiting for new compounds is probably the most relevant [41].

The issue of MDR/XDR and treatment of these cases with new drugs was discussed in several studies. The TB-NET group described the features and outcomes of MDR cases managed in Germany during 2004-2006 [42]. The same group described the efficacy and tolerability of linezolid (used off-label) in treating hopeless MDR-/XDR-TB cases in Italy and Germany in the largest cohort (89 patients) studied to date [43]. A similar study, performed on a much smaller cohort (11 cases), has been performed by a Korean group [44]. A study performed in Tomsk (Russian Federation) by a Russian and US group demonstrated the potential of moxifloxacin in improving the probability of a positive outcome in XDR-TB cases [45]. The relative importance of each XDR-defining drug (first-line drugs including rifampicin and isoniazid, fluoroquinolones and second-line injectable drugs) has been described for the first time by the TBNET. The last study of the series [46] demonstrated that resistance to second-line injectables (and capreomycin in particular) predicts death and failure in MDR-/XDR-TB cases.

\section{CONCLUSIONS}

The promiscuous use of antibiotics in the treatment of LRTIs accounts for a major part of the community burden of antibiotic use and contributes dramatically to the rising prevalence of resistance among major human pathogens. A better understanding of the role of viruses in COPD exacerbations and of new biomarkers in the specific diagnosis of bacterial infection will lead to more focused management of patients with community-acquired LRTIs, thereby resulting in better resource utilisation.

The utilisation of new diagnostic methods for the diagnosis of TB will lead to more specific management of the disease in an environment in which XDR-TB has been reported in 45 countries [47].

Members of the Respiratory Infections Assembly are actively working on these problems, and the results of their research will lead to new approaches to medical knowledge and technologies to improve the management of communityacquired LRTI and TB.

\section{REFERENCES}

1 Woodhead M, Blasi F, Ewig S, et al. Guidelines for the management of adult lower respiratory tract infections. Eur Respir J 2005; 26: 1138-1180.

2 Migliori GB, Besozzi G, Girardi E, et al. Clinical and operational value of the extensively drug-resistant tuberculosis definition. Eur Respir J 2007; 30: 623-626.

3 Matteelli A, Migliori GB, Cirillo D, et al. Multidrugresistant and extensively drug-resistant Mycobacterium tuberculosis: epidemiology and control. Expert Rev Anti Infect Ther 2007; 5: 857-871.

4 Genomics to combat Resistance against Antibiotics in Community-acquired LRTI in Europe. www.grace-lrti.org.

5 Seemungal TA, Harper-Owen R, Bhowmik A, et al. Detection of rhinovirus in induced sputum at exacerbation of chronic obstructive pulmonary disease. Eur Respir J 2000; 16: 677-683.

6 Borg I, Rohde G, Löseke S, et al. Evaluation of a quantitative real-time PCR for the detection of respiratory syncytial virus in pulmonary diseases. Eur Respir J 2003; 21: 944-951.

7 Greenberg SB, Allen M, Wilson J, et al. Respiratory viral infections in adults with and without chronic obstructive pulmonary disease. Am J Respir Crit Care Med 2000; 162: 167-173.

8 Alber D, Quint J, Donaldson G, et al. Quantitative changes in rhinovirus load in exacerbations of COPD. Eur Respir J 2008; 32: Suppl. 52, 510s.

9 Christ-Crain M, Müller B. Biomarkers in respiratory tract infections: diagnostic guides to antibiotic prescription, prognostic markers and mediators. Eur Respir J 2007; 30: 556-573.

10 Christ-Crain M, Jaccard-Stolz D, Bingisser R, et al. Effect of procalcitonin-guided treatment on antibiotic use and outcome in lower respiratory tract infections: cluster-randomised, single-blinded intervention trial. Lancet 2004; 363: 600-607.

11 Lacoma A, Prat C, Domínguez J, et al. Utility of procalcitonin, neopterin, C-reactive protein and MR-pro ANP measurement in the management of exacerbations in chronic obstructive pulmonary disease. Eur Respir J 2008; 32: Suppl. 52, 479s.

12 Maekura R, Kitada S, Hiraga T, et al. Long-term survival of patients with Mycobacterium avium-intracellulare complex 
pulmonary disease (MAC-PD). Eur Respir J 2008; 32: Suppl. $52,395 \mathrm{~s}$.

13 Dautzenberg B, Piperno D, Diot P, et al. Clarithromycin in the treatment of Mycobacterium avium lung infections in patients without AIDS. Chest 1995; 107: 1035-1040.

14 Wallace RJ Jr, Brown BA, Griffith DE, et al. Clarithromycin regimens for pulmonary Mycobacterium avium complex. The first 50 patients. Am J Respir Crit Care Med 1996; 153: 1766-1772.

15 Wallace Jr RJ, Zhang Y, Brown-Elliott BA, et al. Repeat positive cultures in Mycobacterium intracellulare lung disease after macrolide therapy represent new infections in patients with nodular bronchiectasis. J Infect Dis 2002; 186: 266-273.

16 Griffith DE, Aksamit T, Brown-Elliott BA, et al. An official ATS/IDSA statement: diagnosis, treatment, and prevention of nontuberculous mycobacterial diseases. Am J Respir Crit Care Med 2007; 175: 367-416.

17 Richeldi L. An update on the diagnosis of tuberculosis infection. Am J Respir Crit Care Med 2006; 174: 736-742.

18 Hinks T, Thomas M, Raghuraman S, et al. Rapid diagnosis of tuberculous meningitis by enumeration of cerebrospinal fluid antigen-specific T cells. Eur Respir J 2008; 32: Suppl. 52,1 s.

19 Jafari C, Thijsen S, Bossink A, et al. Comparison of BAL ELISPOT and NAT for the diagnosis of smear-negative tuberculosis - a TBNET study. Eur Respir J 2008; 32: Suppl. $52,1 \mathrm{~s}$.

20 Goletti D, Carrara S, Lange C, et al. Response to RD1 selected epitopes is associated to active TB. Results of a European multicentre hospital-based study of the European tuberculosis network (TBNET). Eur Respir J 2008; 32: Suppl. 52, 1s.

21 Carrara S, Butera O, Vanini V, et al. Use of QuantiFERON TB gold in tube to detect LTBI and to monitor prophylaxis in children recently exposed to M. tuberculosis. Eur Respir J 2008; 32: Suppl. 52, 736s.

22 Carrara S, Butera O, Vanini V, et al. An assay based on RD1 selected peptides for the diagnosis of extra-pulmonary and smear negative tuberculosis. Eur Respir J 2008; 32: Suppl. 52,800 s.

23 Ruhwald M, Hiroshi N, Cuevas L, et al. Improving T-cell assays for the diagnosis of latent TB infection: potential of a diagnostic test based on IP-10. Eur Respir J 2008; 32: Suppl. 52,2 s.

24 Ruhwald M, Bodmer T, Maier C, et al. Evaluating the potential of IP-10 and MCP-2 as biomarkers for the diagnosis of infection with Mycobacterium tuberculosis. Eur Respir J 2008; 32: Suppl. 52, 2s.

25 Sester U, Mack U, Fousse M, et al. Flow-cytometric analysis of antigen-specific CD4 T-cell cytokine profiles distinguishes active tuberculosis from successfully treated disease. Eur Respir J 2008; 32: Suppl. 52, 265 s.

26 Carrara S, Butera O, Vanini V, et al. Memory response to RD1 antigens in TST+ subjects: a better tool to detect LTBI? Eur Respir J 2008; 32: Suppl. 52, 2s.

27 Bergamini BM, Losi M, Vaienti F, et al. Performance of blood tests for the diagnosis of latent tuberculosis infection in children. Eur Respir J 2008; 32: Suppl. 52, 1s.

28 Ozere I, Liduma I, Skenders G, et al. Importance of TSPOT.TB test in diagnosing of $M$. tuberculosis infection in
BCG vaccinated children aged five years and younger. Eur Respir J 2008; 32: Suppl. 52, 737s.

29 Bocchino M, Bruzzese E, Matarese A, et al. Performance of IFN- $\gamma$ release assays for tuberculosis detection in immune-compromised children in a low prevalence country. Eur Respir J 2008; 32: Suppl. 52, 798s.

30 Latorre I, Lacoma A, Souza-Galvao MD, et al. IFN-gamma response on T-cell based assays in HIV patients for detection of tuberculosis infection. Eur Respir J 2008; 32: Suppl. 52, 2s.

31 Leidl L, Mayanja-Kizza $H$, Baseke J, et al. Interferon- $\gamma$ release assays for the diagnosis of latent tuberculosis infection in HIV-seropositive individuals. Eur Respir J 2008; 32: Suppl. 52, 799s.

32 Vicidomini S, Bartalesi F, Goletti D, et al. Usefulness of QuantiFERON ${ }_{\mathbb{B}}$-TB gold on LTBI detection in subjects with immunomediated inflammatory diseases. Eur Respir J 2008; 32: Suppl. 52, 737s.

33 Cagatay T, Aydin M, Gulbaran Z, et al. Comparison of PPD and QuantiFERON tests in determining latent tuberculosis in patients who are directed to TNF antagonists. Eur Respir J 2008; 32: Suppl. 52, 800s.

34 De Iaco G, Carvalho AC, Baschè R, et al. Interferon gamma assay for health care workers. Eur Respir J 2008; 32: Suppl. $52,736 \mathrm{~s}$.

35 Christopher DJ, Daley P, Armstrong L, et al. Discordance between T-cell-based assay and tuberculin skin testing among nursing students in India. Eur Respir J 2008; 32: Suppl. 52, 799s.

36 Kobashi Y, Mour K, Obase Y, et al. Clinical utility of the QuantiFERON TB-2G test for elderly patients with active tuberculosis. Eur Respir J 2008; 32: Suppl. 52, 799s.

37 Janssens JP, Roux-Lombard P, Triverio PA, et al. Interferon- $\gamma$ release assays (IGRA) vs. tuberculin skin testing (TST) for detecting latent tuberculosis infection (LTBI) in chronic hemodialysis (CH) patients. Eur Respir J 2008; 32: Suppl. 52, 737s.

38 Diel R, Loddenkemper R, Nienhaus A. Predictive value of a whole-blood IGRA for the development of active TB disease. Eur Respir J 2008; 32: Suppl. 52, 799s.

39 Higuchi A, Kawabe Y, Mitarai S, et al. Comparison of performance in two diagnostic methods for M. tuberculosis infection. Eur Respir J 2008; 32: Suppl. 52, 736s.

40 Migliori GB, Lange C, Centis R, et al. Resistance to secondline injectables and treatment outcomes in multidrugresistant and extensively drug-resistant tuberculosis cases. Eur Respir J 2008; 31: 1155-1159.

41 Migliori GB, Lange C, Girardi E, et al. Fluoroquinolones: are they essential to treat multidrug-resistant tuberculosis? Eur Respir J 2008; 31: 904-905.

42 Eker B, Ortmann J, Migliori GB, et al. MDR- and XDR-TB in Germany. Eur Respir J 2008; 32: Suppl. 52, 228 s.

43 Migliori GB, Eker B, Esselmann A, et al. Efficacy and tolerability of linezolid in the treatment of MDR-TB cases: a TBNET study in Germany and Italy. Eur Respir J 2008; 32: Suppl. 52, 229s.

44 Nam HS, Koh WJ, Park SH, et al. Daily half-dose linezolid for the treatment of intractable multidrug-resistant tuberculosis. Eur Respir J 2008; 32: Suppl. 52, 229s.

45 Tonkel T, Mishustin S, Strelis A, et al. Treatment outcomes of the use of moxifloxacin in management of patients with 
XDR TB in Tomsk Oblast of the Russian Federation. Eur Respir J 2008; 32: Suppl. 52, 227s-228s.

46 Migliori GB, Muetterlein R, Sotgiu G, et al. Second-line injectables: are they essential to treat MDR/XDR-TB cases? Eur Respir J 2008; 32: Suppl. 52, s227.
47 The World Health Organization/International Union Against Tuberculosis and Lung Disease Global Project on AntiTuberculosis Drug Resistance Surveillance, Anti-Tuberculosis Drug Resistance in the World. Report No. 4. Report no. WHO/ HTM/TB/2008.394. Geneva, World Health Organization, 2008. 